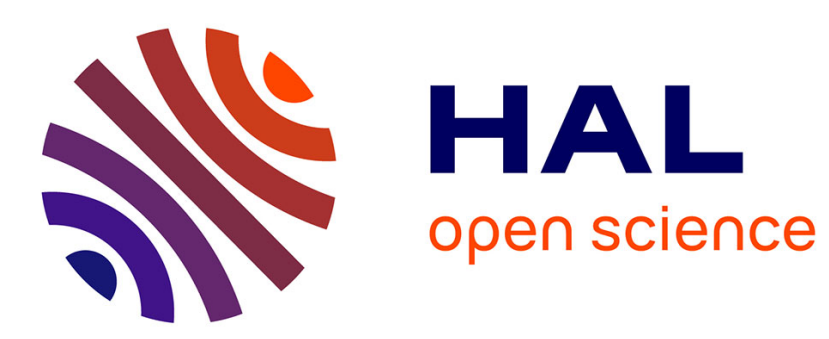

\title{
Heat transfer within combusting droplets
}

Guillaume Castanet, Fabrice Lemoine

\section{To cite this version:}

Guillaume Castanet, Fabrice Lemoine. Heat transfer within combusting droplets. Proceedings of the Combustion Institute, 2007, 31, pp.2141 - 2148. 10.1016/j.proci.2006.07.043 . hal-01570417

\section{HAL Id: hal-01570417 https://hal.univ-lorraine.fr/hal-01570417}

Submitted on 29 Jul 2017

HAL is a multi-disciplinary open access archive for the deposit and dissemination of scientific research documents, whether they are published or not. The documents may come from teaching and research institutions in France or abroad, or from public or private research centers.
L'archive ouverte pluridisciplinaire HAL, est destinée au dépôt et à la diffusion de documents scientifiques de niveau recherche, publiés ou non, émanant des établissements d'enseignement et de recherche français ou étrangers, des laboratoires publics ou privés. 


\title{
Heat transfer within combusting droplets
}

\author{
G. Castanet ${ }^{a}$, F. Lemoine ${ }^{b, *}$ \\ ${ }^{a}$ Technische Universität Darmstadt, Fachgebiet Strömungslehre und Aerodynamik, \\ Petersenstraße 30, 64287 Darmstadt, Germany \\ b LEMTA-CNRS UMR 7563, 2 Avenue de la Forêt de Haye, BP 160 F-54504 Vandouvre-les-Nancy Cedex, France
}

\begin{abstract}
The improvement of the basic understanding of heat transfer in sprays is a key point in many engineering applications. In this paper, the temperature field within combusting ethanol droplets in linear stream is investigated by the two-color laser induced fluorescence technique. Additionally, a heat transfer model within the droplet is developed, taking into account both heat conduction and heat advection by the droplet internal fluid circulation, according to the Hill vortex pattern. Heat and mass exchanges between the liquid and the gas phases are described within the framework of the quasi-steady approach and the film theory. Comparisons between measurements and computational results allow determining the intensity of the Hill vortex related to the maximum velocity at the droplet surface. An expression of the friction coefficient for combusting and interacting droplets is derived from the case of an isolated droplet and a good agreement with the experimental data is observed.
\end{abstract}

Keywords: Droplets; Evaporation; Combustion; Temperature measurements; Heat and mass transfers

\section{Introduction}

The spray and the droplets are central problems in the internal combustion engines. In engines, like turbojets, or direct injection Diesel engines for automobiles, the fuel is introduced into the combustion chamber by high pressure atomizers as a liquid spray. As a fuel droplet enters in a high temperature environment, like in a combustion chamber, the droplet is heated, evaporates and finally the fuel vapor burns to release heat. The quality of the predictive tools in that field is highly related to the level of physical knowledge of the different elementary phe-

\footnotetext{
* Corresponding author. Fax: +33 383595544 .

E-mail address: fabrice.lemoine@ensem.inpl-nancy.fr (F. Lemoine).
}

nomena. Most of the complex phenomena can be captured on basic experiments based on droplets arrays which have been widely used in either experimental or theoretical studies. A few numerical studies (e.g. [1]) have highlighted that the heat transfer within a droplet was the results of combined conduction and advection due to the formation of internal vortexes, caused by the friction phenomena between the liquid droplet surface and the external air flow. The internal velocity field given by the Hill vortex model is usually used to describe such a situation within a moving droplet and a correlation of the friction coefficient was proposed in [2], in the case of a moving evaporating isolated droplet. The robustness of this correlation has never been tested for interacting droplets in combustion, which is a first step in the modeling of the complex heat and mass transfer occurring in real spray flames. The scope of the 


\begin{tabular}{|llll|}
\hline \multirow{2}{*}{ Nomenclature } & \\
$a$ & thermal diffusivity & \multicolumn{2}{l|}{ Greek symbols } \\
$B_{\mathrm{M}}$ & mass transfer Spalding number & $\lambda$ & thermal conductivity \\
$C$ & non dimensional distance parameter & $\rho$ & specific density \\
& $(C=L / D$, with $L:$ droplet spacing) & $\mu$ & dynamic viscosity \\
$D$ & droplet diameter & \multicolumn{2}{l}{ Subscripts } \\
$L_{\mathrm{V}}$ & liquid fuel latent heat of vaporization & amb lambient conditions in the flame \\
$N u$ & Nusselt number & $g$ & gas phase \\
$P$ & pressure & $l$ & fuel liquid phase \\
$R e$ & droplet Reynolds number & $\mathrm{K}$ & fuel \\
$S h$ & Sherwood number & $\infty$ & free stream conditions \\
$Y_{\mathrm{K}}$ & fuel vapor mass fraction & \multicolumn{3}{l}{} \\
\end{tabular}

present paper is to use experimental results about the temperature field within combusting droplets to derive the vortex intensity, when drop to drop interactions are present. The analysis of the within droplets temperature field was carried out using two-colors laser induced fluorescence $([3,4])$. The principles of the within droplet heat transfer modeling will be detailed in the present paper, in order to compute the droplet internal temperature field. The vortex intensity will be adjusted to fit optimally the experimental data. Finally, the evolution of the mean droplet temperature will be computed and compared to experimental test cases.

\section{Experimental set-up}

\subsection{Generation of combusting monodisperse drop- lets stream}

A linear monodisperse droplet stream is generated by Rayleigh disintegration of a liquid jet $([3,5])$, with the use of a mechanical vibration obtained by a piezoceramic. For some given frequencies, the liquid jet breaks up into equally spaced and monosized droplets (Fig. 1). The fuel can be pre-heated in the injector body by means of an external temperature regulated water circulation. The temperature of the fuel is measured at the injection point with the use of thermocouple. An electrically heated coil allows initiating the combustion just after the break-up zone of the liquid jet and creates a laminar continuous column shaped flame. This kind of flame shape is observed for all the droplet spacing tested in the present study.

\subsection{Droplet temperature mapping using two-color laser induced fluorescence}

The details of the technique can be found in a previous paper [5]. The fuel, ethanol here, is previously seeded with a low concentration $(1.2 \mathrm{mg} / \mathrm{l})$

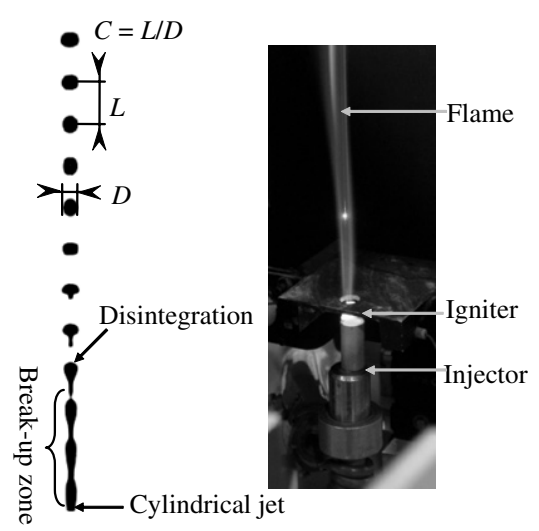

Fig. 1. Picture of the monodisperse droplet stream in combustion.

of rhodamine $\mathrm{B}$, which is an organic dye usually used as a fluorescent temperature sensor. Furthermore, the fluorescence of rhodamine B can be easily induced by the green line $(\lambda=514.5 \mathrm{~nm})$ of the argon ion laser. It requires the detection of the fluorescence signal on two spectral bands for which the temperature sensitivity is highly different. This ratio of the fluorescence signals measured on the two spectral bands appears only temperature dependent and the dependency in added fluorescent tracer concentration, probe volume dimensions, laser intensity and optical layout is totally removed.

If the probe volume is larger than the droplet to provide a global excitation of all the droplet volume and if the signal is averaged on all the droplet transit in the probe volume, a volume averaged droplet temperature can be obtained [5]. The possibility of combusting droplets temperature mapping was reported in two previous papers $([3,4])$ and only the main principles are summed up in the present development. As a droplet crosses the laser excitation area in the stream direction, if the probe volume is small compared to the droplet, different zones of the droplet are successively 
illuminated by the laser and emit fluorescence, which can be processed by the two-colors LIF technique. By shifting the probe volume in a perpendicular axis of the stream direction, it is possible to scan different lines in the droplet. Taking into account the lensing effect of the air-droplet interface, the probe volume dimensions are roughly $(20 \times 20 \times 57 \mu \mathrm{m})$. The main difficulty is to localize the zone of measurement within the droplet, affected by the refraction of the laser beam at the air-droplet interface. A criterion based on the excitation intensity is used, since the contribution of a designated area to the total collected fluorescence signal is proportional to the local laser excitation intensity [4]. The local excitation intensity is determined by a simplified geometrical optics model $([3,6])$, validated by a rigorous approach using generalized Lorentz-Nie theory [4]. The accuracy of the technique is within $\pm 1{ }^{\circ} \mathrm{C}$ and the relative error is mainly due to the probe volume positioning inaccuracy, estimated around $10 \mu \mathrm{m}$ [3].

\section{Modeling of the heat transfer within the droplet}

The temperature $T$ within a droplet, taking into account the effect of the internal liquid circulation and surface regression due to evaporation is governed by the equation:

$$
\frac{\partial T}{\partial t}-\frac{r^{*}}{R} \cdot \frac{\mathrm{d} R}{\mathrm{~d} t} \frac{\partial T}{\partial r^{*}}+\frac{1}{R} \vec{V} \cdot \vec{\nabla} T=\frac{a}{R^{2}} \Delta T
$$

In Eq. (1), $r^{*}=r / R$, where $R$ is the time varying droplet radius. It will be assumed that the internal streamlines of the liquid circulating within the droplet follows a spherical Hill vortex pattern, according to stream function $\psi[7]$ :

$$
\psi\left(r^{*}, \theta, \varphi\right)=-\frac{U_{\mathrm{s}} R^{2}}{2} r^{* 2}\left(1-r^{* 2}\right) \sin ^{2} \theta
$$

where $\left(r^{*}, \theta, \varphi\right)$ are the spherical coordinates of a point within the droplet. According to Sirignano [8], this flow pattern should remain still valid in the case of interacting droplets; however, the drag coefficient, the Nusselt and Sherwood numbers are likely to be modified.

As a consequence of Eq. (2), the maximum surface velocity at the droplet surface $U_{\mathrm{S}}$ may be estimated as $U_{\mathrm{S}}=\frac{1}{6 \pi}\left(V_{\infty}-V\right)\left(\frac{\mu_{g}}{\mu_{l}}\right) \operatorname{Re} C_{\mathrm{F}}$. [2]. The friction coefficient $C_{\mathrm{F}}$ suggested in [2], in the case of an isolated evaporating droplet, is:

$$
C_{\mathrm{F}}=\frac{12.69}{\operatorname{Re}^{2 / 3}\left(1+B_{\mathrm{M}}\right)}
$$

This expression, found for isolated evaporating droplets, should be reconsidered for interacting and combusting droplets. Since droplets evolve in the wake of previous droplets, the shear stress at the surface is reduced and similarly to the drag coefficient, a strong decrease of the friction coefficient is expected. The liquid phase properties are estimated at the droplet average temperature. The physical properties of the gas phase are evaluated at the reference state $\left(T_{\text {ref }}\right.$ and $\left.Y_{\mathrm{K}, \text { ref }}\right)$ according to the " $1 / 3$ rule" $[9], T_{\text {ref }}=$ $T_{\mathrm{S}}+\left(T_{\mathrm{amb}}-T_{\mathrm{S}}\right) / 3$ and $Y_{\mathrm{K}, \mathrm{ref}}=Y_{\mathrm{K}, \mathrm{S}}+\left(Y_{\mathrm{K}, \mathrm{amb}}-\right.$ $\left.Y_{\mathrm{K}, \mathrm{S}}\right) / 3$. The fuel vapor fraction $Y_{\mathrm{K}, \mathrm{S}}$. is determined, assuming the liquid-vapor equilibrium, by the Clausius-Clapeyron law [10].

Equation (1) is solved under initial condition $T(r, t=0)=T_{\text {inj }}$ supplemented by the conditions at the droplet surface $2 \pi R^{2} \lambda_{l} \int_{\theta=0}^{\pi}\left(\frac{\partial T}{\partial r}\right)_{r=R} \sin \theta$ $\mathrm{d} \theta=Q_{\mathrm{L}}(t)$, where $Q_{\mathrm{L}}$ is the heat flux penetrating into the droplet by conduction and convection from the gas phase.

The instantaneous heat flux $Q_{\mathrm{L}}$ is evaluated by means of the overall energy budget equation, neglecting the radiative exchanges [11]

$Q_{\mathrm{L}}=\Phi_{\mathrm{C}}-\Phi_{\mathrm{vap}}$

where $\Phi_{\mathrm{C}}=\frac{N u \lambda_{g} \pi D}{4}\left(T_{\mathrm{amb}}-T_{\mathrm{S}}\right)$ is the convective heat flux exchanged with the gaseous environment and $\Phi_{\text {vap }}=L_{\mathrm{v}} \pi D \rho_{g} D_{g} B_{\mathrm{M}} S h$ is the heat flux due to vaporization. The Nusselt number $N u$ and Sherwood number $S h$ follow the film theory [2]. In such a configuration, the influence of the droplet to droplet interactions should be taken into account, since the reduced distance parameter $C$ is on the order of a few units. It is known that the decrease of the droplet spacing leads to a decrease of the Nusselt and Sherwood numbers [12]. Therefore, due to similarities between the heat and mass transfer, it was shown that the effects of the interaction are similar on both Nusselt and Sherwood numbers, and may be described by applying the same reduction factor $\eta(C)$, depending only on the reduced droplet spacing $C([10,13])$

$\eta(C)=\frac{S h}{S h_{\text {iso }}}=\frac{N u}{N u_{\text {iso }}}$

where $N u_{\text {iso }}$ and $S h_{\text {iso }}$, defined in [2], represent the Nusselt and Sherwood numbers for an isolated, moving evaporating droplet. The correlation suggested in [10] for $2.5 \leqslant C \leqslant 16$, obtained in the case of combusting monodisperse droplets in linear stream, was used.

The resolution of Eq. (1) is performed by splitting the temperature on the Legendre polynomials base [14]. The equations are solved as proposed by Abramzon and Sirignano [2], taking into account regulation mechanisms, linking the penetrating heat flux $Q_{\mathrm{L}}$, the surface temperature $T_{\mathrm{S}}$, the fuel vapor fraction at the droplet surface $Y_{\mathrm{K}, \mathrm{S}}$, and the vaporization rate $\dot{m}$. An increase in the heat flux penetrating into the droplet involves an increase of the droplet surface temperature which causes a rise of the surface fuel vapor fraction and thus finally an increase of the evaporation rate, which tends to limit the initial increase of heat flux. 


\section{Experimental results and comparison with mod- eling}

\subsection{Within droplet temperature distribution}

Two cases of monodisperse droplet streams, characterized by different droplet diameters, velocities and droplet spacing at the injection point were investigated. The different injection parameters of the three test cases are summarized in Table 1.

The droplet velocity was measured by LDV, in order to evaluate the time elapsed from the injection point and the values of the measured velocity were used in the calculation.

The temporal evolution of the within droplet temperature field, corresponding to the first test case $(D=216 \mu \mathrm{m})$ is presented in Fig. 2, at three time steps. It appears clearly that the heat transfer is not purely radial, the temperature distribution resulting partially from advection caused by the internal circulation. It can be noticed that the heat is transported from the lateral sides of the droplet to its wake side, and then to the center, which agrees qualitatively with the Hill vortex description. The comparison to the liquid phase heat transfer model is now possible if the ambient temperature $T_{\mathrm{amb}}$ is known. In a previous studies conducted in similar experimental conditions [10], the ambient temperature was ranging between 800

Table 1

Aerothermal parameters of the investigated monodisperse droplet streams in combustion

\begin{tabular}{lccccc}
\hline$D_{0}(\mu \mathrm{m})$ & 216 & 130.6 & 104.8 & 223 & 90.7 \\
$D_{\mathrm{f}}^{*}(\mu \mathrm{m})$ & 213.8 & 127.4 & 96.4 & 211 & 75 \\
$T_{\text {inj }}\left({ }^{\circ} \mathrm{C}\right)$ & 35.4 & 40.5 & 36 & 40 & 34.3 \\
$V_{0}(\mathrm{~m} / \mathrm{s})$ & 9.4 & 8.1 & 8.2 & 9 & 5.8 \\
$C$ & 3.5 & 5 & 4.2 & 4.4 & 7.7 \\
$K^{*}$ & 1.5 & 2 & 1.7 & 1.7 & 1.7 \\
$T_{\text {amb }}^{*}\left({ }^{\circ} \mathrm{C}\right)$ & \multicolumn{1}{c}{850} & 900 & 900 & 850 & 900 \\
\hline
\end{tabular}

* Adjusted parameters; index " 0 ", first measurement point; $D_{\mathrm{f}}$, final diameter in the period of investigation. and $1100{ }^{\circ} \mathrm{C}$ and was determined using the measured liquid phase temperature at the equilibrium. In the present, the ambient temperature was not investigated and remains still unknown in the range $\left[800{ }^{\circ} \mathrm{C} ; 1100{ }^{\circ} \mathrm{C}\right]$. Different simulations have shown that the spatial arrangement of the isothermal lines was not significantly influenced by the ambient temperature contrary to the vortex intensity. However, it is clear that the ambient temperature modifies mainly the surface temperature. Then, it appears possible to perform in first the adjustment of the vortex intensity and subsequently a more refined adjustment of the ambient temperature, the influence of the adjusted temperature on the isothermal lines arrangement, in the investigated range, remaining very low. Consequently, the vortex intensity was adjusted in first and the ambient temperature in second.

In order to improve the validity of the comparison with the experimental results, the averaging effect of the optical set-up on the calculation results is taken into account as done in [4]. Consequently, as for the experimental data, the area close to the surface is not visible and a low dissymmetry between the left and right parts of the temperature maps, caused by the effect of the optical set-up, can be noticed.

Since the maximum surface velocity requires certainly being adapted for combusting and interacting droplets, it is proposed to modify the friction coefficient defined in Eq. (3) and to replace its expression by the more general form:

$$
C_{\mathrm{F}}=\frac{K}{R e^{2 / 3}\left(1+B_{\mathrm{M}}\right)}
$$

where $K$ is a parameter adjustable in the light of the experimental data.

In order to test the sensitivity of the parameter $K$, the within droplet temperature field was calculated for various values of the maximum surface velocity $U_{\mathrm{S}}$ and for the same value of the ambient temperature, fixed at $850{ }^{\circ} \mathrm{C}$. The influence of the parameter $K$ alone can be observed in Fig. 3,

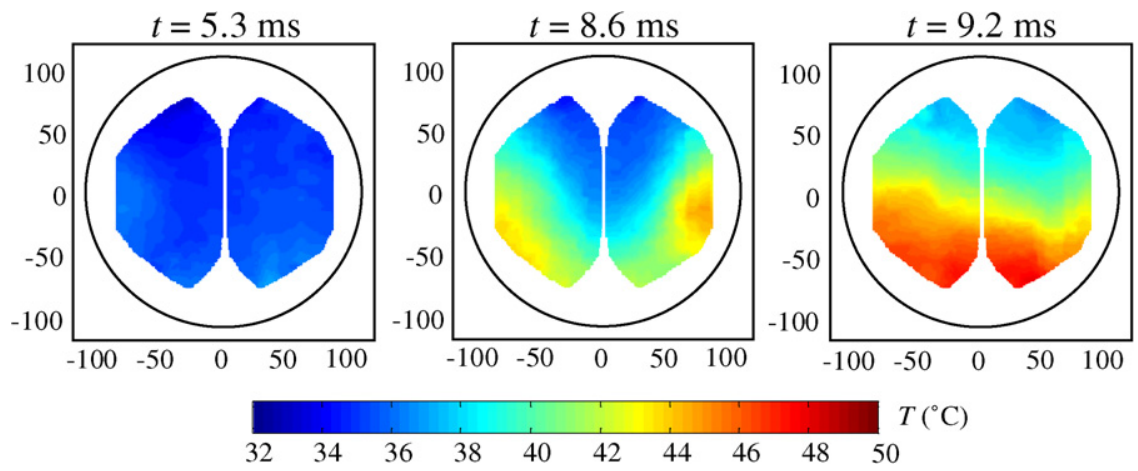

Fig. 2. Temperature maps at different successive time of the combustion (color code is in ${ }^{\circ} \mathrm{C}$, horizontal and vertical scales are in microns, $D=216 \mu \mathrm{m}, C=3.5$ ). 
where only the last experimental instant is represented $(t=9.2 \mathrm{~ms})$. As seen in Fig. 3, the value $K=12.69$ is clearly not adapted and the heat diffusion speed appears too important for $K=2$ or 2.5 , since a hot stream located in the droplet center can be observed on the simulations and not for the experimental temperature maps. For a lower value of $K$, for example $K=1$, the heat advection becomes restricted and the isothermal lines tend to match with pure radial conduction. Finally, for the intermediate value $K=1.5$, the arrangement of the isothermal lines and the distribution of the hot and cold zones within the droplet seem consistent with the experiment. In the light of the comparison between the experimental results and numerical simulations, the expected accuracy on the determination of $K$ is better than 0.5 . The influence of the ambient temperature was also studied separately. The selection of $T_{\mathrm{amb}}=850^{\circ} \mathrm{C}$ $\left( \pm 50^{\circ} \mathrm{C}\right)$ seems to fit well with the experiments. Experimental temperature fields and numerical simulations of different time steps of the droplet heating phase have been compared for the selected value of $K=1.5$ (Fig. 4), for $D=216 \mu \mathrm{m}$ : a good agreement between the temporal evolution of the temperature maps and the numerical calculations is observed. To get further validations of this analysis, the same study was carried out on a smaller droplet diameter $(D=131 \mu \mathrm{m}$, see Table 1$)$. The value of the parameter $K$, as well as the ambient temperature have been adjusted independently, as proposed in the preceding analysis, to obtain the optimal agreement with the measured within droplets temperature fields (Fig. 5). The results, for the two test cases are gathered in Table 1 . The value of $K$ seems to range between 1.5 and 2 , which is compatible with the uncertainty on the determination of this parameter. For the value $K=1.7$, the quadratic discrepancy averaged on the overall temperature map reaches a minimum value of about $1.9^{\circ} \mathrm{C}$.

\subsection{Mean droplets temperature evolution}

The predictions of the previously detailed model combined with the adjusted value of the parameter $K$ have been tested in the light of mean droplets temperature measurements for different conditions of combusting monodisperse droplet streams (Table 1). The ambient temperature $T_{\mathrm{amb}}$ used in these simulations is determined with the help of the measured equilibrium temperature, using the method proposed by Castanet et al. [10]. The four test cases presented in Fig. 6, for injection droplet diameter ranging from 90.7 to $223 \mu \mathrm{m}$ and different distance parameters, confirm that the value $K=1.7$ is correctly adapted to describe the droplet heating.

\section{Concluding remarks}

The heat transfer within combusting monodisperse droplets streaming linearly has been investi-
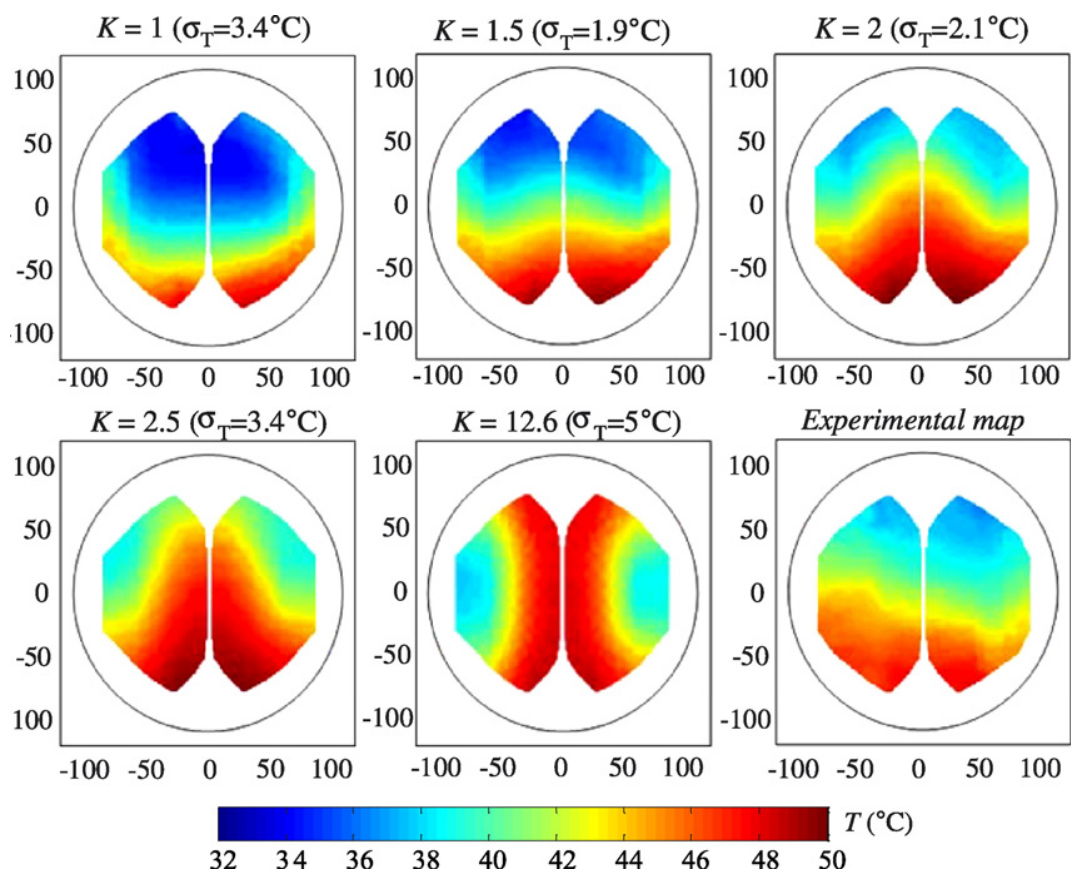

Fig. 3. Influence of the parameter $K$ on the spatial arrangement of the isothermal lines, $D=216 \mu \mathrm{m}, t=9.2 \mathrm{~ms}$, $T_{\mathrm{amb}}=850^{\circ} \mathrm{C}$ (horizontal and vertical scales are in microns, $\sigma_{\mathrm{T}}$ is the quadratic discrepancy averaged on the overall temperature map). 

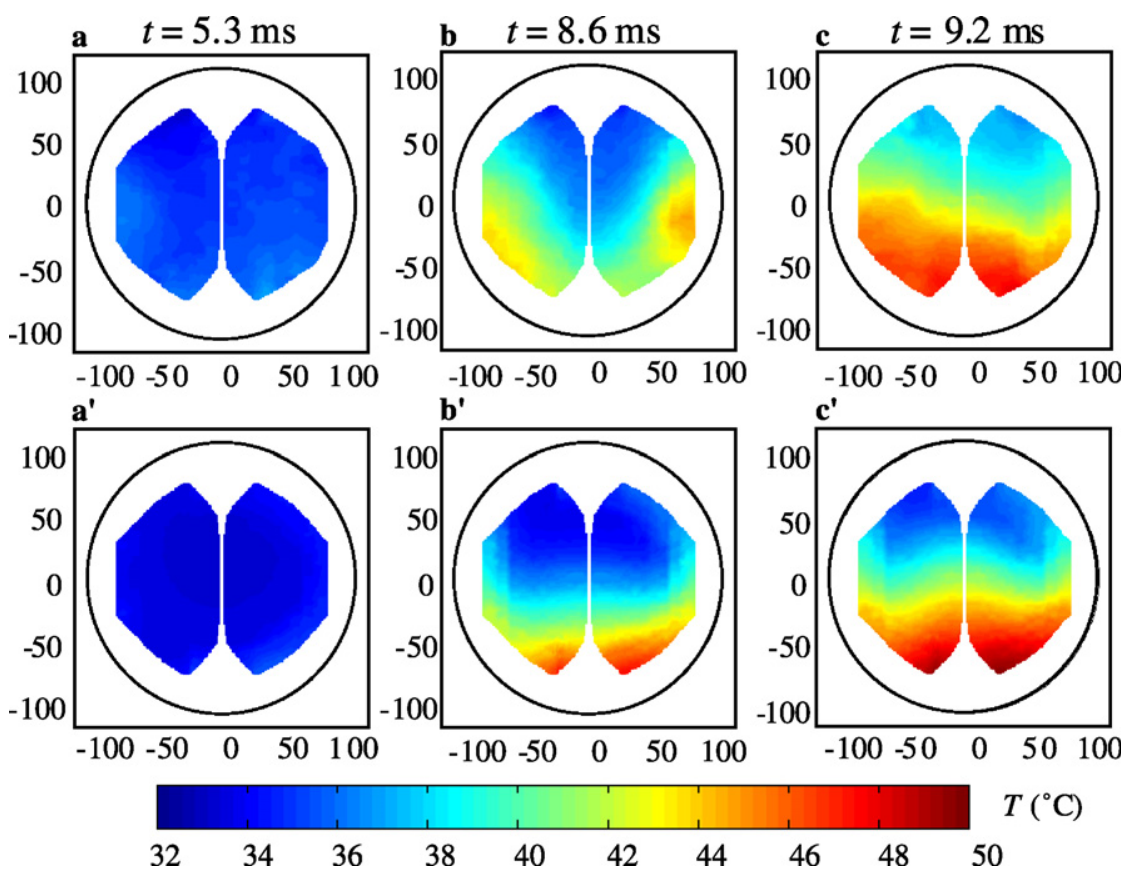

Fig. 4. Comparison between the numerical simulations and the experiments, $D=216 \mu \mathrm{m}, C=3.5, T_{\mathrm{amb}}=850{ }^{\circ} \mathrm{C}$, $K=1.5$ (horizontal and vertical scales are in microns, a-c, experimental maps; $\mathrm{a}^{\prime}-\mathrm{c}^{\prime}$, simulation results).
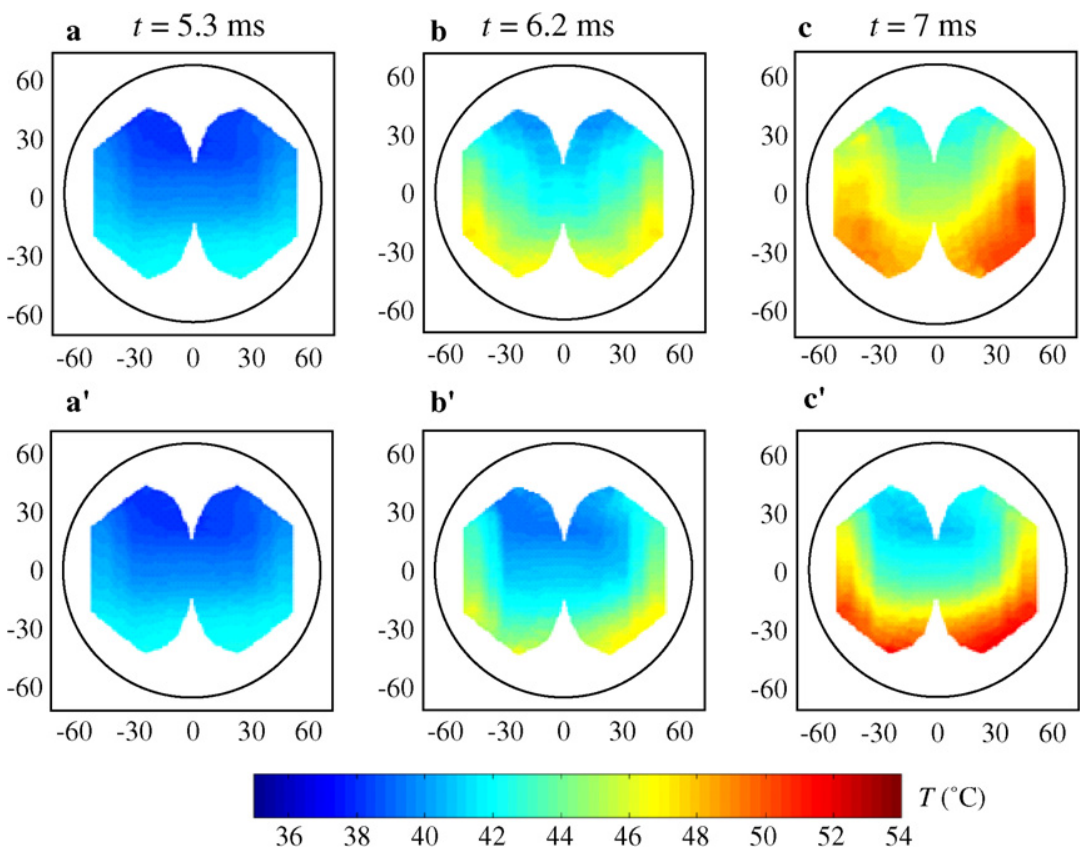

Fig. 5. Comparison between the numerical simulations and the experiments, $D=130.6 \mu \mathrm{m}, \mathrm{C}=5, T_{\mathrm{amb}}=900{ }^{\circ} \mathrm{C}$, $K=2$ (horizontal and vertical scales are in microns, a-c, experimental maps; $\mathrm{a}^{\prime}-\mathrm{c}^{\prime}$, simulation results).

gated with a simplified model in parallel with an experimental analysis.

On the one hand, the model is based on heat advection within the droplets by the Hill vortex system. It has been demonstrated that the vortex intensity, related to the friction coefficient at the droplet interface, has a strong influence on the within droplets temperature pattern. On the other 


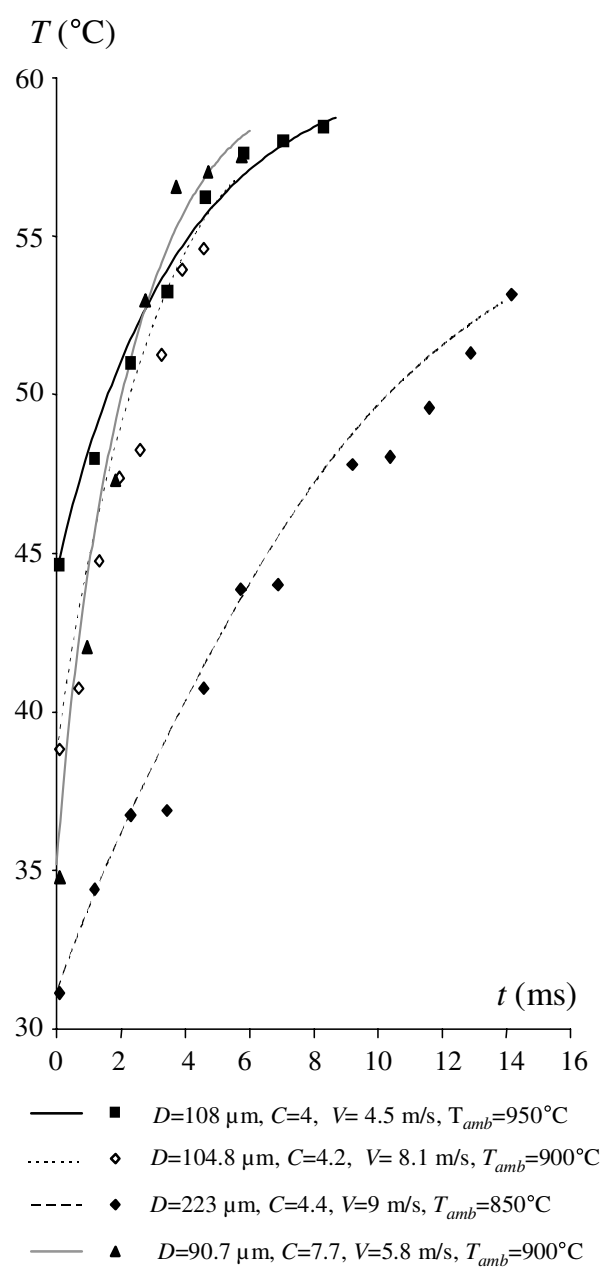

Fig. 6. Mean droplet temperature measurements of combusting droplets streaming linearly and comparison with the simulation (parameter $K$ adjusted to 1.7). (Lines, fitted curves; dots, experimental data.)

hand, experimental maps of the within droplets temperature at different time of the combustion, for two test cases have been constructed.

A comparison between the calculated temperature fields within the droplets using the simplified heat transfer model and the experimental temperature maps allows determining the friction coefficient with a reasonable accuracy. This coefficient may be derived from the expression suggested by Abramzon and Sirignano [2], i.e. $C_{\mathrm{F}}=\frac{K}{R e^{2 / 3}\left(1+B_{\mathrm{M}}\right)}$, but the value of $K=12.69$ proposed by these authors must be significantly decreased for interacting and combusting droplets $(K=1.7)$. The comparison of the measured temporal evolution of the mean droplet temperature and calculations using the adjusted value of the friction coefficient appears reliable to assess the adjustment of the parameter $K$. Because frictions are mainly related to the flow pattern around the droplet stream, this result can be used in many situations where a main flowfield direction is present. The friction coefficient is likely to vary with the inter-droplet spacing; however, no variation can be clearly pointed out from the experimental data which correspond to a relatively narrow range of interdroplet spacing.

\section{Acknowledgment}

This work is supported by the European Community in the framework of the MUSCLES contract, Growth project GRD1-2001-40198.

\section{References}

[1] C.H. Chiang, M.S. Raju, W.A. Sirignano, Int. J. Heat Mass Transf. 35 (1992) 1307-1324.

[2] B. Abramzon, W.A. Sirignano, Int. J. Heat Mass Transf. 32 (1989) 1605-1618.

[3] G. Castanet, P. Lavieille, M. Lebouché, F. Lemoine, Exp. Fluids 35 (2003) 563-571.

[4] G. Castanet, A. Delconte, F. Lemoine, L. Mees, G. Gréhan, Exp. Fluids 39 (2005) 431-440.

[5] P. Lavieille, F. Lemoine, G. Lavergne, M. Lebouché, Exp. Fluids 31 (2001) 45-55.

[6] P. Lavieille, F. Lemoine, G. Lavergne, J.F. Virepinte, M. Lebouché, Exp. Fluids 29 (2000) 429-439.

[7] R. Clift, J.R. Grace, M.E. Weber, Bubbles, Drops, and Particles, Academic, New York, 1978.

[8] W.A. Sirignano, Fluid dynamics and transport of droplets and sprays, Cambridge University Press, 1999.

[9] G.L. Hubbard, V.E. Denny, A.F. Mills, Int. J. Heat Mass Transf. 18 (1975) 1003-1008.

[10] G. Castanet, M. Lebouché, F. Lemoine, Int. J. Heat Mass Transf. 48 (2005) 3261-3275.

[11] T. Elperin, B. Krasovitov, Int. J. Heat Mass Transf. 38 (1995) 409-422.

[12] C.H. Chiang, W.A. Sirignano, Int. J. Heat Mass Transf. 36 (1993) 875-886.

[13] J.F. Virepinte, Y. Biscos, G. Lavergne, P. Magre, G. Collin, Combust. Sci. Tech. 150 (2000) 143-159.

[14] G. Castanet, P. Lavieille, M. Lebouché, F. Lemoine, Combust. Sci. Tech. 177 (2005) 2395-2422. 